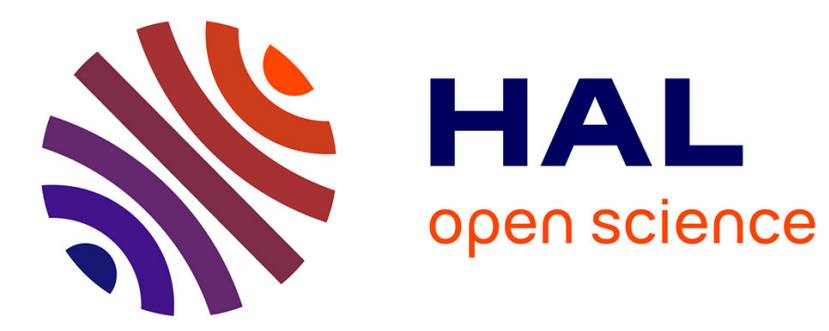

\title{
Itinéraires du corps augmenté: déficiences et performances dans le sport
}

\author{
Isabelle Queval
}

\section{To cite this version:}

Isabelle Queval. Itinéraires du corps augmenté: déficiences et performances dans le sport. Corps \& psychisme : recherches en psychanalyse et sciences , 2020, 1 (76), pp.21-32. hal-03044298

\section{HAL Id: hal-03044298 \\ https://hal-inshea.archives-ouvertes.fr/hal-03044298}

Submitted on 7 Dec 2020

HAL is a multi-disciplinary open access archive for the deposit and dissemination of scientific research documents, whether they are published or not. The documents may come from teaching and research institutions in France or abroad, or from public or private research centers.
L'archive ouverte pluridisciplinaire HAL, est destinée au dépôt et à la diffusion de documents scientifiques de niveau recherche, publiés ou non, émanant des établissements d'enseignement et de recherche français ou étrangers, des laboratoires publics ou privés. 


\section{Itinéraires du corps augmenté : déficiences et performances dans le sport}

\section{Le sport de haut niveau : un laboratoire expérimental de l'amélioration humaine}

Parce qu'il porte à leur extrême, si ce n'est à leur excès, nombre d'attitudes contemporaines concernant la performance humaine, le sport de haut niveau est un laboratoire expérimental, mais aussi une éprouvette du social. Tout d'abord il est un laboratoire expérimental du dépassement de soi, mais cette notion a presque une connotation mythifiée, tant elle est liée au vécu subjectif et au romanesque de l'effort. Plus prosaïquement, le sport de haut niveau procède aujourd'hui d'une anthropotechnie qui vise à scientifiser et techniciser l'amélioration de l'humain. La démarche est holistique, chaque paramètre de la performance et chaque détail de la vie de l'athlète étant pris en compte dans cette programmation/fabrication : matériels et matériaux, techniques gestuelles et science de l'entraînement, suivi médical, diététique, psychologique, protocoles de récupération, éventuellement de complémentation biologique et physiologique, etc. Dans les sports professionnels, par équipes ou individuels, les mieux dotés, comme le football ou le tennis par exemple, les data viennent compléter la panoplie des «staffs ». Il s'agit alors de prévoir et assortir la performance d'un environnement statistique très sophistiqué, qui vise à réduire à tout coup la fameuse, et soi-disant «glorieuse », incertitude du sport.

La continuité scientifique entre les protocoles de préparation/récupération et le dopage est à ce titre évidente même s'il existe une discontinuité morale et législative entre le permis et le défendu. Repérons que depuis les années 2000, des voix ont contesté l'argument moral de la lutte anti-dopage (principalement la rupture de l'équité entre les compétiteurs), mais aussi suggéré qu'un dopage médicalisé, c'est-à-dire contrôlé, en quelque sorte officiel et encadré, servirait plutôt qu'il ne desservirait la santé des athlètes ${ }^{1}$. Cette posture, si elle n'est pas (encore) majoritaire, conforte néanmoins l'idée qu'en ces temps où la performance sportive est annoncée comme physiologiquement stagnante à l'orée des années $2020^{2}$, le spectacle sportif et ses enjeux pourraient perdurer dans le futur grâce au renforcement de diverses formes de dopage, chimique et pharmacologique, mais aussi génétique et technologique, seules à même de permettre encore l'amélioration des performances.

\section{L'idéologie sportive emblématise un phénomène social diffus et d'ampleur variable : les « attitudes dopantes »}

L'idéologie sportive, spécifiquement celle du sport de haut niveau mais pas que, nourrit l'imaginaire de l'augmentation. Rappelons que l'histoire du sport est aussi celle de l'inventivité technique, parfois extravagante. Le matériel sportif, quel qu'il soit, concentre des enjeux scientifiques et économiques qui attestent l'impact social, médiatique, géopolitique même de la performance. L'artifice s'hybride avec la naturalité du corps pour mener l'athlète toujours «plus vite, plus haut, plus fort », selon la formule. La médecine, la pharmacologie, la diététique participent de ce processus par lequel l'aliment ou le médicament sont comme un «entrânement invisible » ${ }^{3}$. Typiquement, le champion est toujours en recherche de ce «petit plus » qui lui permettra de prendre l'avantage sur ses concurrents.

Certes, on peut souligner que « performer ", au fond, est aussi une manière de s'arracher à soi, à l'instant, à l'immédiat, au statu quo, parfois pour mieux se trouver, s'accomplir, s'élever, mais pas toujours. Lorsque les sportifs «s'éclatent» ou «se déchirent», il y a toujours le risque d'un épuisement physique et/ou psychique, d'une fuite en avant, à toujours vouloir « faire mieux ». Cette problématique, qui n'est pas propre au sport, a été pointée dès les années 1990 par les critiques du « culte de la performance » comme leitmotiv social ${ }^{4}$. La différence, toutefois, entre la sphère du sport

\footnotetext{
${ }^{1}$ Bengt Kayser, Alex Mauron, Andy Miah, Legalisation of performance-enhancing drugs, The Lancet, 366, 2005 , p. 521 ; B. Kayser, A. Mauron, A. Miah, Current anti-doping policy : a critical appraisal, bmc Medical Ethics,2007, 8, p. 2.

${ }^{2} \mathrm{Cf}$. Les travaux du Pr. Jean-François Toussaint à l'IRMES.

${ }^{3}$ L'expression est de Philippe Lucas, lorsqu'il entraînait la nageuse Laure Manaudou.

${ }^{4}$ Cf. Les travaux d'Alain Ehrenberg notamment.
} 
de haut niveau et d'autres sphères sociales comme celles de l'entreprise ou de l'école, c'est que le contre-discours, celui qui met en garde contre l'excès des pratiques et cite les dangers associés, ne peut être entendu. En effet, le sport de haut niveau est fondé, ontologiquement, sur le culte de la performance. Il est une course aux records depuis ses origines compétitives, ou il n'est pas. Par-là, il demeure un lieu privilégié au sein duquel étudier ce que signifie «l'augmentation de l'humain à visée de performance » à l'époque contemporaine.

Dans cet esprit, le sport, même celui du sportif « ordinaire», est le terreau d' «attitudes dopantes ». Ces dernières, sans être légalement répréhensibles comme le dopage, consistent à être dépendant de l'idée qu'on ne peut être «performant»-en sport, au travail, à l'université, dans la préparation d'un examen, dans une prise de parole publique ou tout simplement parfois en se levant le matin- qu'en consommant des produits « optimisants ». C'est une culture de l'adjuvant qui traverse notre société. Du jus d'orange aux amphétamines, du café aux médicaments «à usages sociaux détournés » tels le Modafinil ou la Ritaline, en passant par les compléments alimentaires, les antidépresseurs ou les drogues dites « récréatives », le spectre est large de ces consommations plus ou moins banales ou banalisées, censées favoriser la performance. Le sportif qui, comme le rappelle Claire Carrier, est obsédé par « le boire et le manger » dans un but de performance, manifeste une « dépendance à l'oralité » qui le rend particulièrement vulnérable aux «attitudes dopantes » ${ }^{5}$, et emblématique d'un glissement de sens entre bien-être et mieux-être, santé et performance observé ailleurs dans la société.

\section{Une nouvelle anthropotechnie du corps interroge les notions de « corps naturel » et de « corps artificiel »}

Quelques corps sportifs contemporains posent en outre une question inédite, celle de la limite du corps humain «naturel » et de l'avantage « artificiel » procuré par les prothèses. Longtemps tapie dans l'ombre de la hiérarchie jusque-là indiscutable des performances, séparant «valides » et « handicapés », cette question est aujourd'hui en pleine lumière, depuis que des athlètes avec prothèses, dont le plus connu est Oscar Pistorius, approchent tout près des records des «valides ». L'avantage supposé que procure, ou pourrait procurer à l'avenir, ces prothèses, interroge non seulement sur la règle de la compétition sportive et l'établissement de ses catégories, sur la notion de « dopage technologique », mais aussi plus avant sur le rapport du corps humain à la technique, et sa transformation ou « augmentation » par la science. Car si la prothèse devenait un réel avantage sportif, nul doute que la pression concurrentielle du sport d'élite pourrait conduire à se mutiler des champions désireux de bénéficier d'un tel avantage. Les représentations du corps « naturel » ou « artificiel » sont ici en jeu, plus précisément la question des contours, c'est-à-dire aussi de l'identité de l'humain, tandis qu'est omniprésent, dans la littérature ou le cinéma, l'imaginaire du cyborg. Mais la réflexion éthique doit aussi s'engager sur l'instrumentalisation des corps et l'atteinte à l'intégrité des individus. Comment penser son identité, s'approprier son corps, pas seulement le corps de la performance, mais celui du désir et des émotions, le « corps propre » ou «moi corporel » selon le concept de MerleauPonty, si la dépendance technique crée une étrangeté à soi-même ? Quid d'une éthique de l'autonomie si se développe cette emprise, et cette entreprise, anthropotechnique?

\section{L'émergence d'un questionnement}

Dans les années 2000, en franchissant le premier la barre des 11 "au $100 \mathrm{~m}$ dans la catégorie «T44» («simple amputé tibial»), l'athlète américain Marlon Shirley, par ailleurs sauteur en longueur et en hauteur, a contribué à introduire le débat sur le rôle des prothèses dans la performance sportive. De manière inédite, depuis l'avènement du sport olympique, un athlète amputé et concourant avec une prothèse a porté ses performances à un niveau soutenant la comparaison avec le sport valide. Même si les écarts demeurent importants d'un point de vue sportif, ils sont minimes sur le plan humain et questionnent dès lors l'équivalence entre les performances du sport et celles de l'handisport. Ce questionnement prend une ampleur médiatique internationale à partir de 2004, lorsque le sud-

\footnotetext{
${ }^{5}$ Cf. Claire Carrier, L’adolescent champion, contrainte ou liberté, PUF, 1992.
} 
africain Oscar Pistorius («double amputé tibial», mais concourant dans la catégorie «T44 »), bouscule tous les records établis dans le sport paralympique et tutoie les sommets des performances internationales du 400m «valides ». Quelques années plus tard, médaillé aux jeux paralympiques, Pistorius fait la demande d'une participation aux compétitions « valides » lors des jeux olympiques de Pékin en 2008. Cette demande est tout d'abord rejetée par la Fédération Internationale d'Athlétisme (IAAF), puis finalement acceptée. Sans rentrer dans la controverse de spécialistes, on reproche à ses prothèses notamment de « grandir » Pistorius, et de lui permettre de courir le second $200 \mathrm{~m}$ aussi vite que le premier, ce que ne font pas ordinairement les sportifs valides. Finalement, en 2012, aux jeux Olympiques de Londres, Pistorius participera en tant que remplaçant du relais 4X400m sud-africain «valides », en sus des médailles collectées aux jeux paralympiques.

Or ce nous qui intéresse ici, c'est le terme d' « avantage » employé pour motiver le refus initial. On rappellera tout d'abord que l' " avantage » est ce qui est recherché par définition par la compétition sportive et, en particulier, tel que l'atteste l'histoire des sports, par l'évolution des techniques et matériels depuis 150 ans. Dès lors, si une évolution est validée on peut l'appeler un "progrès », si elle est rejetée, son usage entraînera une « tricherie ». Ainsi, dans son registre, pour l'instant chimique et pharmacologique, le dopage constitue une recherche d' « avantage », certes prohibée pour deux motifs principaux, la contrevenance au principe d'égalité des chances et l'atteinte à la santé des athlètes, mais qui ne s'en inscrit pas moins dans une démarche globale d'amélioration. L'éventail des moyens propres à gagner un « avantage » est ainsi étendu et il appartient à la règle sportive de trancher entre le permis et le défendu.

Par conséquent, il paraît donc peu probant de rejeter les prothèses de Pistorius au seul motif qu'elles constituent un « avantage ", si on considère que tous les paramètres de l'entraînement sportif visent cet objectif, et sauf à qualifier cet « avantage » de « dopage technologique », tel qu'on l'a fait à propos des combinaisons intégrales de natation, ou à imaginer l' «augmentation» du corps comme un progrès technique.

\section{La prothèse comme « avantage artificiel » ou « dopage technologique » ?}

Or, précisément, apparait pour la prothèse le même argument employé pour le dopage, celui du recours à un «avantage artificiel », redoublant l'usage confus des notions. Car, de même que l'« avantage » est ce qui est recherché communément par le sport, de même ce dernier se définit, dès l'origine, comme une activité «technique », «technicienne », c'est-à-dire au sens philosophique « artificielle », selon l'opposition aristotélicienne entre la " nature » et l' " artifice ». L'artifice est ce qui est créé par l'homme, comme par exemple la technique. En ce sens, le dopage apparaît certes comme une « amélioration artificielle » de la performance » (amélioration chimique), mais au même titre qu'une combinaison de natation (« dopage technologique»), une roue lenticulaire (progrès technique) ou une prothèse de jambe (dispositif médical), à ceci près qu'il altère la santé des athlètes. Si les notions d' " avantage » ou d' "artifice» ne sont pas déterminantes pour (dis-)qualifier la spécificité des prothèses, et condamner de facto leur utilisation dans les compétitions «valides », en revanche l'image d'un corps à la fois « handicapé » et «performant» bouscule les représentations. D'une part, elle illustre la transition qui s'opère de la déficience à la performance et ouvre de nouvelles voies pour penser le handicap. D'autre part, la thématique sportive rejoint une thématique plus globale qui est celle de l'«amélioration», - enhancement- en anglais ou encore de l' «augmentation », dont l'une des figures de proue est l'idéologie transhumaniste. C'est ainsi que la réparation des corps par des dispositifs prothétiques ou numériques, et surtout la compétitivité de ces corps dans le sport engagent une réflexion inédite sur le « corps augmenté » et le «sport augmenté ».

\section{De la déficience à la performance}

Oscar Pistorius, comme Aimée Mullins, ancienne athlète et désormais mannequin néerlandaise alternant une douzaine de paires de prothèses de jambes en fonction de ses activités, ne se revendiquent, ni même ne se considèrent comme « handicapés ». Égéries de marques de mode -ce n'est plus le cas pour Pistorius, à présent incarcéré pour le meurtre de sa petite amie- ils ont effectivement transformé leur déficience initiale - nés sans péronés, tous deux ont été amputés à l'âge 
de 11 mois- en un « avantage » social, si ce n'est sportif. La prothèse est leur image de marque, la clé de voûte de leur reconnaissance publique. Par là, ils initient un changement radical de perspective sur le handicap et son rapport à la performance sportive et sociale, et popularisent la notion de supercrip. Ainsi, pour les disabilities studies (les études d'influence anglo-saxonne sur le handicap), le supercrip est un individu en situation de handicap qui démontre une capacité au-delà de celles communément attendues pour les personnes handicapées, une telle capacité pouvant s'exprimer dans de nombreux domaines. Force est de constater que la prouesse physique est le registre le plus spectaculaire et le plus problématique. Dans le sport de haut niveau, le supercrip invite en particulier à repenser la catégorisation selon trois axes principaux, mêlant les aspects sportif, social et philosophique.

On remarquera tout d'abord que d'autres champions avant Pistorius avaient déjà concouru à la fois lors des Jeux Paralympiques ou des Deaflympics (réservés aux sourds), et des Jeux Olympiques, sans que leur situation n'ait provoqué de remous dans la presse ou bousculé les règlementations. À chaque fois, la hiérarchie des catégories avait en quelque sorte été respectée puisque les performances des «handicapés » étaient inférieures à celles des «valides». Aussi, le cas Pistorius est avant tout singulier en ce que ses performances sont susceptibles de dépasser celles des valides. On imagine à juste titre que des prothèses de jambes, ou autres, pourraient permettre à l'avenir de battre des records «toutes catégories ». On évoque le « dopage technologique». On questionne la règle sportive. On observe que la technologie des prothèses d'un « double amputé tibial » permettra des progrès dont ne peuvent bénéficier les «simples amputés tibiaux », limités de fait par leur jambe «naturelle ». Un sportif « plus handicapé » pourrait donc battre à la course un autre «moins handicapé ». En 2014, le sauteur en longueur allemand Markus Rehm, vainqueur des championnats nationaux, est interdit de championnat d'Europe quelques semaines plus tard, au motif que sa prothèse l' «avantage » par rapport aux valides.

\section{Le handicap, entre « opposition » et « variation »}

Une tension inédite traverse donc la notion de catégorie sportive et sa pertinence quand il s'agit d'appréhender les nouvelles performances des sportifs équipés de prothèses, et déborde sur le champ social s'agissant des représentations du handicap. Comme le remarque très justement Pierre Ancet $^{6}$, «nous pensons par oppositions (ce qui est humain, ce qui ne l'est pas; ce qui vaut la peine, ce qui n'en vaut pas la peine; ce qui est normal, ce qui ne l'est pas), comme pour lutter contre l'existence d'un continuum entre les différents termes de ces alternatives. [...] La réalité peut être perçue différemment : l'anormal peut être vu comme une variation sur le thème de la normalité. Il peut être vu comme une autre forme de normalité, une différence plus qu'une anormalité, une autre manière de vivre et d'exister, non moins riche, non moins valorisable. [...] La véritable normalité est adaptation et création, ce dont on peut être profondément incapable tout en étant socialement normal. » Le plus souvent, les mesures issues des normes médicales et des normes sociales, et de l'interaction entre les deux, tiennent en réalité peu compte de la capacité humaine à se dépasser, à produire une originalité ou à être productrice de normes, y compris physiologiques ou psychologiques. D'autant que ces normes sont toujours contextualisées. L'existence de cette potentialité créatrice, que Canguilhem appelle «normativité », nous invite donc à penser par «variations » plutôt que par «oppositions ».

Les performances de Pistorius ou Rehm troublent les conventions, les perceptions du corps humain, et questionnent sur cette simple «différence de degrés », cette «variation » comme dit Canguilhem, séparant la performance « handicapée » de la performance « valide ». Elles questionnent aussi logiquement sur la nature de l'«inclusion» de sportifs tels que les supercrips dans les compétitions valides : est-ce une manière de reconnaître le handicap via l'inclusion et la visibilité, ou au contraire de le nier en effaçant la déficience au profit de la performance ? La catégorie compétitive, ancrée dans une histoire ancienne du sport, peut-elle résister aux problèmes sociétaux posés par la technologisation de l'humain, mais aussi, par la réflexion sur le genre, et la «différence de degrés »

\footnotetext{
${ }^{6}$ Pierre Ancet, «Les savoirs de la philosophie », in Charles Gardou (dir.), Handicap, une encyclopédie des savoirs, Erès, 2014, p. 193-208.
} 
entre le féminin et le masculin ? Est-ce une représentation normative du corps, et donc de l'humain, de ses contours et limites, qui doit trancher parmi les utilisations possibles d'exosquelettes?

Comme on le voit, la question du « dépassement» par le sportif handicapé des performances « valides », cette inversion de valeurs et de représentations ouvre à des questions inédites sur le corps « augmenté » qui dépassent très largement le cadre du sport.

\section{Questions éthiques et philosophiques}

Le handicap étant défini comme le marqueur individuel et social d'une incapacité, comment évoquer une "surcapacité » concernant les supercrips ? Comme le précise Jérôme Goffette, deux registres de légitimité sont en parallèle concernant le corps «augmenté »: le médical et l'anthropotechnique. Les deux perspectives reposent sur des finalités -ou des téléologiesfoncièrement différentes, et susceptibles d'entrer en conflit. Dans le cas de Pistorius, utilisant deux sortes de prothèses, pour la vie courante et pour le sport, la perspective médicale a en somme fourni deux réponses, et conforte la légitimité des prothèses. Dans la perspective anthropotechnique, la finalité n'est plus la santé, mais la performance et la compétition, et alors les prothèses doivent être soumises à l'examen technique susceptible de déceler un avantage « déloyal » (unfair advantage) constitutif d'une tricherie.

Mais plus avant, la question du corps «augmenté » évoque l'imaginaire du cyborg ou de compétitions opposant des Robocop du sport. La science-fiction, dans la littérature et le cinéma, regorge de ces scénarios où s'affrontent davantage des dispositifs techniques et des multinationales que des humains, dans des compétitions entre super héros figurant le sport. "L'être humain n'est alors que le module de base, dit Goffette, sur lequel s'emboîte le dispositif spécifique à telle ou telle performance, à la façon de ces outils modulaires des bricoleurs sur lesquels on peut adjoindre différentes fonctionnalités ${ }^{7} »$.

L'organisation des catégories de la compétition sportive débouche sur une réflexion cruciale. Si on maintient les catégories actuelles qui séparent «handicapés » et «valides », l'exemple de Pistorius ou d'autres, susceptibles de battre des records « valides », conduit cette catégorisation à une impasse, ou à sa suppression pure et simple. Si, conséquemment, on admet les sportifs équipés de prothèses dans toutes les compétitions, et que ces prothèses confirment le gain global de performance, alors on peut envisager, compte tenu des pressions liées à la concurrence que, tôt ou tard, certains compétiteurs valides auraient recours à la mutilation pour rester compétitifs. "La compétition sportive, déjà fortement pathogène au vu des enquêtes sur les effets du dopage, aurait encore accru son emprise sur les corps, encore davantage aliéné les personnes pour les affuter toujours plus affuter des instruments de performance ${ }^{8} »$. Ces mutilations représenteraient alors un extrême dans l'aliénation humaine et l'atteinte portée à l'intégrité.

Pistorius ou Mullins invitent à penser le handicap, ici la déficience physique, comme une force, une source de dépassement, de soi bien sûr, mais aussi des autres et des situations. Comment interpréter cela ? Pour ce qui est de la «prothétisation » des athlètes et de sa catégorisation dans la compétition, il est du ressort de la législation sportive de lui accorder un statut, présent et à venir, qui garantisse l'intégrité physique et morale des personnes. Pour ce qui est d'évaluer l'avenir du handicap à l'aune de ces cas exceptionnels, il faut évidemment rester vigilant quant à la banalisation de ces «augmentations » et la sous-estimation de ce qu'est le quotidien avec une prothèse. Il reste ici à souhaiter que la pression de la compétition ou de la conformité aux standards ne joue aucun rôle moteur, et que l'inflation des prothèses et des modifications puisse être contenue par une attitude de prudence. À force de changements, l'individu risquerait de ne plus se retrouver lui-même ou, dans la dépendance technique accrue, de voir son potentiel d'autonomie altéré. En outre, de même que chacun ne peut devenir un sportif de haut niveau, de même chaque déficience ne peut être transformée en

\footnotetext{
${ }^{7}$ Jérôme Goffette, « Modifier les humains : anthropotechnie versus médecine », in Jean-Noël Missa et Laurence Perbal (dir.), «Enhancement »-Éthique et philosophie de la médecine d'amélioration, Paris, Vrin, 2009, p.62.

${ }^{8}$ Ibid., p.61.
} 
performance. Enfin, concernant l'image renvoyée par ces athlètes, un pas a été franchi, dans la reconnaissance et la médiatisation, mais aussi dans les représentations d'un corps «esthétique » ou « fonctionnel». Selon cette perspective, et bien que tous les «handicaps » ne se valent pas, on peut espérer des bienfaits de l'éclairage porté par le sport sur le handicap, et du cheminement qui s'opère ainsi vers une évolution plus positive et plus universelle de ses représentations.

\section{L'horizon fantasmatique du posthumain}

De toute évidence, l'inflation des prothèses remanie nos conceptions du corps. Alors qu'il était plus facile autrefois de superposer l'entité corporelle d'un corps "fermé » à l'identité personnelle, aujourd'hui le corps « ouvert » de la médecine de greffes et de prothèses fragilise cette identification. Qui suis-je si « tout » en moi est interchangeable et améliorable, si « tout » est matériau et renouvelable, dans une sorte d'apoptose technologique ? Le «corps propre », corps-chair et muscles du sportif, son corps-émotions aussi s'imbrique à un corps machine ou instrument, dont l'ampleur des modifications possibles et de la sujétion à l'emprise économique et politique, est inédite. En outre, certains affirment, comme les transhumanistes, que l'humain performant de demain sera technologisé, «augmenté », tandis que l'humain «naturel » sera «handicapé ». Ceci ne constituerait plus alors un futur de la «perfectibilité » comme " amélioration», mais une rupture, annonçant une «posthumanité ». Le risque est ici, pour l'individu de se perdre, et de perdre son autonomie, comme de perdre l'humain. Face à cet emballement anthropotechnique, il est souhaitable que le souci de la performance sportive n'oblitère pas une indispensable prudence éthique.

\section{L'augmentation : inflation d'un corps-prothèse ou rupture avec l'humain ?}

On pourrait dire au fond que le corps sportif est un corps-prothèse bien avant l'utilisation même de cet objet compensatoire et artificiel, à visée médicale, nommé « prothèse ». Parce qu'il est travaillé, sculpté, exhibé, choyé comme un objet fétiche et de jouissance, le corps du sportif obnubilé par ses fameuses «sensations » est un corps médiateur avec le monde, paradoxalement un corpsbéquille lui qui est producteur de performances, et support du «moi ». Il n'est qu'à voir le corps des culturistes comme représentation extrême de cette condition.

Deux sens de l' « augmentation » viennent s'intriquer l'un à l'autre dans le sport : celui d'une hybridation consubstantielle de l'homme avec la technique qui date de la «nuit des temps » et qui fait de l'homme fondamentalement un être technique; celui d'une amélioration des capacités humaines par l'ajout d'un appareil, d'un artifice, d'un objet technique, c'est-à-dire d'un exosquelette, y compris quand cet objet vient compenser le manque d'une fonction ou d'un membre puisqu'on a vu que le sport paralympique tendait à faire de la prothèse un "avantage compétitif ». Ainsi, les supercrips du sport sont à l'origine de nouveaux questionnements sur le corps sportif. L'image de l'infirmité laisse place à celle de la performance, ouvrant un horizon jadis impensable : celui où les sportifs équipés de prothèses domineraient les «valides » dans les compétitions. Cette perspective ne remet pas seulement en cause la réglementation sportive concernant un «avantage » ou un « dopage technologique », ou bien les catégories compétitives elles-mêmes. Elle convoque la réflexion éthique et philosophique pour définir les contours de l'humain, et penser les effets de la technicisation et de l'« augmentation » du corps.

Car en effet, ces deux sens de l' " augmentation » demeurent dans une certaine continuité qui s'exprime au travers du progrès technique et de son accélération, et illustre la dialectique classique entre «nature » et «culture » : quid de la «nature » et de la «culture » chez un coureur à pied dont les chaussures sont moulées à son pied, chez un nageur en combinaison, chez un cycliste, etc.. et finalement chez un athlète paralympique appareillé depuis la petite enfance ? En revanche, la perspective de créer des cyborgs, des êtres fabriqués par la technoscience à fin de performance, rompt avec une certaine histoire de la technique et de l'humain. Elle rompt avec l'humanisme et, dans cette ambition plus ou moins fantasmatique, aux fondements scientifiques pour l'heure contestables, prospère le transhumanisme. Il ne s'agit plus alors de « progrès » mais de « rupture », ni réellement $\mathrm{d}^{\prime}$ '《 augmentation » mais plus justement de « substitution » du posthumain à l'humain.

S’il est à prévoir ainsi que le déploiement de la technoscience, en faisant du corps un objet 
interchangeable et programmable sur commande, fasse peser des risques sur l'autonomie et l'intégrité de la personne humaine, une vigilance éthique aigue est requise, dans le sport et ailleurs, pour peser et réfléchir les enjeux d'une transformation radicale de ce qui fait l'humain, au profit d'intérêts incertains.

Isabelle Queval

isabelle.queval@inshea.fr

Professeure des universités

INS HEA - Université Paris Lumières

Groupe de recherche sur le handicap, l'accessibilité et les pratiques éducatives et scolaires (EA 7287 GRHAPES)

58, avenue des Landes

92150 SURESNES

Associée au LACI (IIAC/EHESS/CNRS UMR 8177)

105 Boulevard Raspail

75006 Paris 